\title{
Descripción de la metodología aplicada en una investigación cualitativa en Expresión Corporal y Danza: la vida y obra de Patricia Stokoe Description of the methodology applied in a qualitative research in Body Expression and Dance: the life and work of Patricia Stokoe
} Antonio José Cardona Linares, Julio Ángel Herrador Sánchez, África Calvo Lluch Universidad Pablo de Olavide (Sevilla)

Resumen: Los inicios de la Expresión Corporal (EC) han sido y están siendo analizados desde diferentes perspectivas. Este trabajo ha pretendido profundizar en los comienzos acaecidos a lo largo del siglo XX, en donde esta disciplina emergente se nutrió de múltiples fuentes: de lo social, las artes, la educación, la psicología, etc. Patricia Stokoe (1919-1996), dedicó su vida al estudio de la Expresión Corporal-Danza (ECy D). Considerada como una de las iniciadoras en este campo (Kalmar, 2005; Cardona, 2009; Ruano \& Sánchez, 2009). Su obra sigue siendo referente en la actualidad y está presente en gran parte de la producción científica que se genera en esta disciplina. Es la investigación de Cardona (2009), en donde se ha profundizado en el estudio de la vida y la obra de la autora. Exponemos aquí cómo se llevó a cabo dicha investigación cualitativa. Se hizo un tratamiento descriptivo transversal, utilizando el análisis de documentos y las entrevistas. Realizamos más de treinta entrevistas entre Buenos Aires y España. Estableciéndose 4 dimensiones/campos que aportaron estructura y claridad al estudio: análisis de la vida; análisis de las influencias en la vida y la obra; análisis de la obra; ensayo bibliográfico de la obra. Se obtuvieron unos resultados y conclusiones que convierte a Patricia Stokoe en una de las pioneras en la construcción y génesis de la Expresión Corporal.

Palabras clave: Patricia Stokoe, expresión corporal, danza, metodología cualitativa.

Abstract: The beginnings of corporal expression have been and are being analyzed from different perspectives. This work has attempted to delve into the beginnings that occurred throughout the 20th century, when this emerging discipline was nourished by multiple sources: society, arts, education, psychology, etc. Patricia Stokoe (1919-1996), devoted her life to the study of body language-dance. Considered one of the initiators in this field (Kalmar, 2005; Cardona, 2009; Ruano \& Sánchez, 2009), her work continues to be a reference nowadays and it is present in a large part of the scientific production generated in this discipline. It is in the research by Cardona (2009), who carried out a doctoral thesis, where the study of the life and work of the author has been deepened. We present here how this qualitative research was carried out. A cross-sectional descriptive treatment was carried out, using the analysis of documents and interviews. We conducted more than thirty interviews between Buenos Aires and Spain, establishing 4 dimensions / fields that provided structure and clarity to the study: life analysis; analysis of the influences on life and work; analysis of the work; bibliographic essay of the work. We obtained results that make the life and work of Patricia Stokoe one of the pioneers in the construction and genesis of corporal expression.

Keywords: Patricia Stokoe, corporal expression, dance, qualitative methodology.

\section{Introducción}

La figura de Patricia Stokoe (Buenos Aires, 1919San Carlos de Bariloche, 1996) (imagen 1), ha sido considerada como una de las creadoras de la EC (Kalmar, 2005; Cardona, 2009; Ruano \& Sánchez, 2009). Escribió ocho libros (tabla 1) que actualmente siguen siendo manuales de referencia en esta disciplina. Además de otro, en donde aparece entrevistada en relación a la EC, la educación por el arte y los procesos de creativos. Su obra es motivo de investigación por diferentes autores:

Fecha recepción: 29-09-21. Fecha de aceptación: 12-01-22

Antonio José Cardona Linares

acardona1969@hotmail.com
Kalmar (2005), Cardona (2009), Ruano \& Sánchez (2009) y García (2015).

Para Peña yVicente (2019) que citan a Bernal (2016):

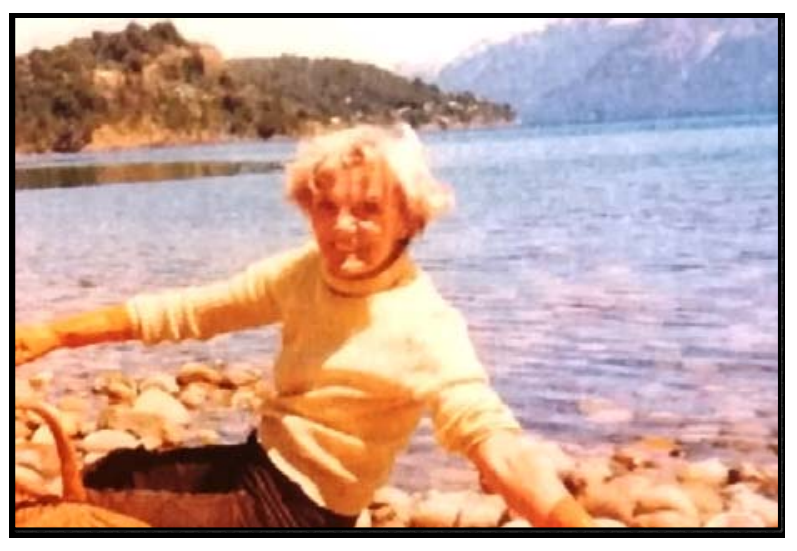

Imagen 1. Patricia Stokoe. (Península San Pedro en San Carlos de Bariloche, Argentina- 1994) 
Tabla 1

Libros publicados por Patricia Stokoc

\begin{tabular}{|c|c|c|c|}
\hline Libro & Autor/es & Año de publicación & Editorial/lugar \\
\hline Expresión corporal y el niño & Stokoe, P. & $1967 / 1982$ & Ricordi: Buenos Aires \\
\hline $\begin{array}{l}\text { Expresión corporal y el adolescente. } \\
\text { (Con fotos de Alex Shächter). }\end{array}$ & Stokoe, P. & 1974 & $\begin{array}{l}\text { Barry: Buenos Aires/ } \\
\text { Ricordi }\end{array}$ \\
\hline La Expresión corporal. & $\begin{array}{l}\text { Schächter, A. y } \\
\text { Stokoe, P. }\end{array}$ & 1977 & Paidós: Buenos Aires \\
\hline $\begin{array}{l}\text { Expresión corporal, guía didáctica para } \\
\text { el docente. }\end{array}$ & Stokoe, P. & $1978 / 1986$ & Ricordi: Buenos Aires \\
\hline $\begin{array}{l}\text { Patricia Stokoe: diálogo con Violeta } \mathrm{H} \text {. } \\
\text { de Gainza. }\end{array}$ & $\begin{array}{l}\text { H. de Gainza, } \\
\text { V. y Stokoe, P }\end{array}$ & 1979 & Lumen: Buenos Aires \\
\hline $\begin{array}{l}\text { Expresión corporal en el jardín de } \\
\text { infantes }\end{array}$ & $\begin{array}{l}\text { Harf, R. y } \\
\text { Stokoe, P. }\end{array}$ & 1980 & Paidós: Buenos Aires \\
\hline $\begin{array}{l}\text { Expresión } \\
\text { educación. }\end{array}$ & Stokoe, P. & $1987 / 1990$ & Lumen: Buenos Aires \\
\hline El proceso de creación en el arte. & $\begin{array}{l}\text { Sirkin, A. y } \\
\text { Stokoe, P }\end{array}$ & $1994 / 1995$ & $\begin{array}{l}\text { Almagesto: Buenos } \\
\text { Aires }\end{array}$ \\
\hline $\begin{array}{l}\text { Educación y expresión estética. } \\
\text { (Entrevista realizada a Patricia } \\
\text { Stokoe). }\end{array}$ & Herbón, A. & 1978 & $\begin{array}{l}\text { Plus Ultra: Buenos } \\
\text { Aires }\end{array}$ \\
\hline
\end{tabular}

«la danza no es indiferente a la educación, es una herramienta que promueve la cultura y la formación de la personalidad y otras dimensiones». Patricia Stokoe dedicó su vida y obra al fomento e integración de la ECy $\mathrm{D}$ en las diferentes facetas de la personalidad humana: cognitiva, física, cognitiva, social, emocional.

Encontramos numerosas citas a la obra de Patricia Stokoe, cuando se hace referencia a la EC, tanto en tesis doctorales, como en artículos de revistas de relevancia científica y es citada por profesorado y doctores que imparten la asignatura de ECy D. A modo de ejemplo de tesis doctorales y artículos: Cachadilla (2004), en su tesis doctoral, cita tres libros de Stokoe. Por otro lado, Ruano (2004), cita cuatro libros de la autora en su tesis doctoral. En los números 15, 23 y 24 de la revista: «Retos. Nuevas tendencias en Educación Física, Deporte y Recreación», Stokoe es citada por autores estudiosos de la EC, como: Baena, Granero, \& Gómez (2009); Coterón \& Sánchez (2013); Calvo, García \& Pérez (2013) y Armada, González \& Montávez, (2013). También en la «Revista Emasf: Revista Digital de Educación Física», en los números 14, 49, 56, Stokoe también es citada por diferentes autores como Coterón \& Sánchez (2012); Montávez (2012); Abilleira \& Fernández (2017) y De la Peñas \& Gil (2019). Por otro lado Padilla, Pérez, \& Fernández (2012), la citan en su trabajo dedicado a la EC en el mundo universitario.

Las hijas de Patricia Stokoe: Deborah Kalmar y Leslie Kalmar que continúa con el legado de su madre, inauguraron el «I Congreso Internacional de Expresión Corporal y Educación» celebrado en Zamora, con sendas ponencias dedicadas a la vida y obra de su madre, tituladas: Con los ojos del corazón (Kalmar D., 2003) y Expresión, Creatividad y Movimiento (Kalmar L., 2003).

Sin embargo pensamos que no ha habido una investigación que profundizará y sistematizara la vida y obra de la autora. De esta forma Cardona (2009), ha centra- do su investigación, como profesional de la EC en la figura de Patricia Stokoe, así su tesis doctoral: «Patricia Stokoe: vida y obra. La creadora de la Expresión Corporal-Danza» (imagen 2). Para desarrollar su trabajo utilizó una metodología cualitativa, en dónde se sirvió de la «entrevista» y «análisis de documento». Entrevistó en Argentina a más de treinta personas relacionadas con la vida y obra de Patricia Stokoe que dieron soporte a las conclusiones que obtuvo en dicha tesis doctoral. Más adelante y dándole visibilidad a este trabajo, publica un libro con la hija de Patricia, Déborah Kalmar, titulado: «La vida de Patricia Stokoe» (Cardona \& Kalmar, 2011).

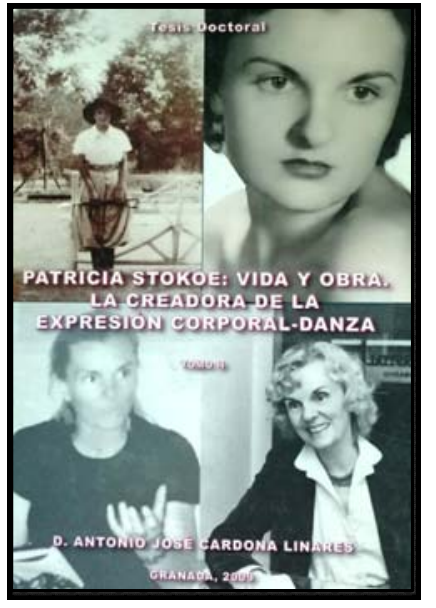

Imagen 2. Portada de la tesis doctoral: "Patricia Stokoe: vida y obra la creadora de la Expresión CorporalDanza". Universidad de Granada, 2009

\section{Objetivos de la investigación}

Los objetivos principales de este este trabajo han sido:

1.- Describir la metodología cualitativa que se llevó a cabo en una investigación en Expresión Corporal y Danza sobre la vida y la obra de Patricia Stokoe.

2.- Exponer algunos de los resultados más relevantes de esa investigación en relación a la vida y obra de Patricia Stokoe.

\section{Material y método}

Nuestro estudio lo consideramos una investigación cualitativa y descriptiva, y más concretamente vinculada hacia el campo de la etnografía, ya que su objetivo es recoger y analizar información fiable, a través de un proceso sistemático, para tomar decisiones sobre un problema de investigación (Latorre, Del Rincón \& Arnal, 2003). Denzin \& Lincoln (2000) definen a la investigación cualitativa como una actividad que sitúa al investigador en el mundo y que consiste en una serie de prác- 
ticas interpretativas que hacen al mundo visible. Se han utilizado dos técnicas para obtener información: el análisis de documentos, relativos a la vida y obra de Patricia Stokoe y las entrevistas personales.

Uno de los procedimientos más utilizados en la investigación cualitativa para garantizar la validez de los datos y la fiabilidad de las interpretaciones es la triangulación (Arroyo, 2005). Para Gillham (2000), es un procedimiento que pretende aproximarse metodológicamente, desde distintos puntos de vista, a un mismo objeto de estudio previamente definido.

Fases del Diseño de investigación: El diseño de la investigación se ha desarrollado en nueve fases (tabla 2). Se centró en profundizar en la vida y la obra de Patricia Stokoe. Para obtener dicha información hemos utilizado como técnica la entrevista. Esta tiene un carácter flexible por ser el resultado de una interacción verbal entre entrevistado y el entrevistador. Cuando nos referimos a la entrevista se habla de una técnica en la cual mediante un proceso de comunicación se pretende obtener información sobre un tema o problema determinado (Santos, 1990). De acuerdo con Visauta (1989), la entrevista es un método de investigación científica, que utiliza un proceso de comunicación verbal para recoger unas informaciones en relación con una determinada finalidad. Las entrevistas se han realizado en un ambiente tranquilo, con tiempo suficiente y de forma individual (Jones, 1985).

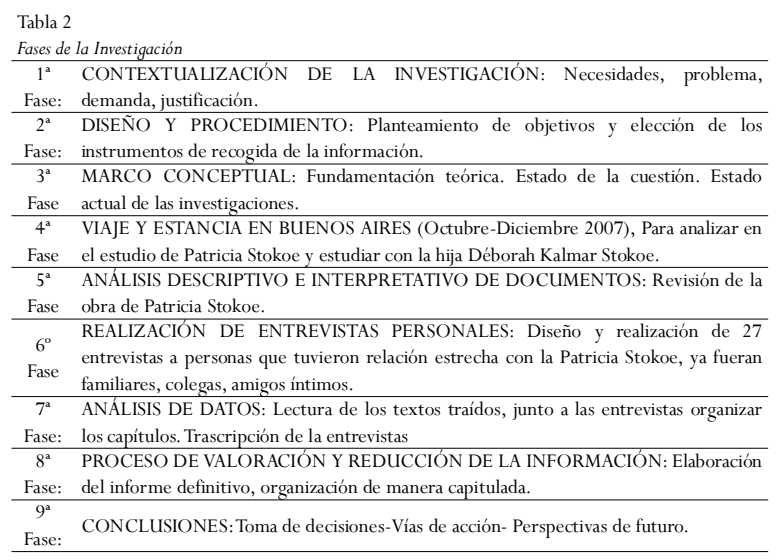

El proceso que se ha seguido para la realización de las entrevistas ha sido el siguiente: en primer lugar se elaboró un protocolo de entrevista, con la persona que consideramos más conocedoras de la vida y obra de Patricia Stokoe: su hija Déborah Kalmar Stokoe. Déborah continúa con la labor de su madre en Buenos Aires, además de haber publicado un libro sobre la obra de Patricia titulado: «Qué es la Expresión Corporal. A partir de la corriente de trabajo creada por Patricia
Stokoe» (2005). Una vez pasado este protocolo de entrevista a dos personas (sujeto 1 y 2), nos sirvió para comprobar la adecuación de la misma o realizar las modificaciones pertinentes, así como para perfilar y orientar la actuación del entrevistador durante el desarrollo de las entrevistas. Tras corregir las carencias observadas, se elaboró la entrevista final que se pasó al resto de sujetos. Una vez finalizadas se pasó a su transcripción y codificación.

Las entrevistas se realizaron a las personas elegidas por Déborah Kalmar, en casa de los entrevistados, y en el IUNA (Instituto Universitario de la Artes- Buenos Aires). Se trató siempre que la situación fuera lo más natural posible. Se pretendió encontrar lo que era importante y significativo: descubrir acontecimientos y dimensiones subjetivas de las personas tales como creencias, pensamientos, valores, etc. (Buendía, Colás \& Hernández, 1998).

La finalidad ha sido obtener información, opiniones, conocimientos sobre la vida y la obra de Patricia Stokoe. Desde el punto de vista de la forma definimos nuestra entrevista como semiestructurada. Al hacerse de forma individual adquiere este carácter ya que hay mayor flexibilidad y libertad de exponer las ideas, aunque su contenido, declaración y secuenciación están en manos del entrevistador (Cohen \& Manion, 1990). A la vez que establece unos elementos diferenciadores que la alejan del término entrevistas conversacionales acuñado por Goetz \& LeCompte (1988).

Para la confección del guión de la entrevista hemos seguido la propuesta de Spradley (1979), que diferencia tres tipos generales de cuestiones: descriptivas, estructurales y de contraste. Como esquema básico de la entrevista hemos seguido la propuesta de Aguirre (1995). Este autor señala tres espacios: 1) Presentación y toma de contacto: donde se presenta la finalidad de la misma, se explica el guión y se busca un clima distendido. 2) Cuerpo de la entrevista: se intenta hacer un planteamiento que va desde cuestiones globales a cuestiones más específicas. 3) Cierre: se repasan aspectos pendientes, y los agradecimientos por la colaboración prestada.

Con el empleo de este tipo de entrevista hemos pretendido: conocer las creencias, pensamientos y Conocimientos sobre la vida y obra de Patricia Stokoe y profundizar en ella. Para la elaboración de las preguntas hemos seguido la tipología establecida por Palomares (2003) \& Torres (2008): preguntas sobre formación inicial, centro, edad y la experiencia profesional. Así como preguntas sobre conocimientos, opiniones y valores, referidas a su pensamiento sobre la figura de nuestra 
protagonista.

Dentro del proceso de investigación se ha realizado un informe que dará como resultado las ideas más importantes de nuestra investigación cualitativa, y se realizó un análisis conjunto de las entrevistas, tratando cada uno de los campos y preguntas de modo transversal. Así este escrito fue un elemento de valoración para la comprensión de la realidad por los sujetos participantes en el contexto estudiado (Mercado, 2000).

La redacción del informe se ha realizado de manera transversal, es decir se han fijado los campos de análisis y se ha introducido información en dicho de campo procedente de todas las entrevistas realizadas, teniendo en cuenta las pretensiones formuladas en los objetivos. Las entrevistas fueron grabadas y posteriormente transcritas. En la investigación interpretativa hemos tenido en cuenta las opiniones expresadas por autores con un papel relevante dentro de este campo de estudio tales como: Taylor \& Bogdan (1987), Colás \& Buendía (1994), Miles \& Huberman (1994) y Stake (1998).

Enumeramos en la (tabla 3) a las personas entrevistadas, según el orden cronológico, el lugar y la fecha de dicha entrevista, así como su vínculo con Patricia Stokoe. También se obtuvo información relevante para esta investigación a partir de las respuestas recibidas por correo electrónico de otras personas relacionadas con Patricia Stokoe (tabla 4). Las personas entrevistadas dieron su consentimiento informado para la futura publicación de dichas entrevistas.
Tabla 4

\begin{tabular}{|c|c|c|c|}
\hline & $\begin{array}{l}\text { Personas entrevistadas } \\
\text { correo electrónico }\end{array}$ & Lugar y Fecha & $\begin{array}{c}\text { Vínculo con } \\
\text { Patricia Stokoe/Dedicación }\end{array}$ \\
\hline 1 & Sujeto 28 & Febrero de 2009 & $\begin{array}{l}\text { Amiga de P.S. Su padre falleció en el mismo kayac } \\
\text { que el marido de Patricia. }\end{array}$ \\
\hline 2 & Sujeto 29 & Febrero de 2009 & $\begin{array}{l}\text { Doctor } / \text { Profesor en } \text { expresión Corporal. } \\
9 \text { Coorganizador del } 1^{\circ} \text { y } 2^{\circ} \text { Congreso internacional de } \\
\text { Expresión Corporal en Zamora }(2003-2008) \text {. }\end{array}$ \\
\hline 3 & Sujeto 30 & Marzo de 2009 & $\begin{array}{l}\text { Amiga y alumna de P.S. Tiene un estudio de } \\
\text { Expresión Corporal en Mar de Plata (Argentina). }\end{array}$ \\
\hline 4 & Sujeto 31 & Abril de 2009 & $\begin{array}{l}\text { Músico, pianista improvisador en el Collegium } \\
\text { Musicum y en el estudio de Patricia (del primer } \\
\text { grupo de trabajo). }\end{array}$ \\
\hline
\end{tabular}

Aunque las entrevistas que se hacían eran semiabiertas, exponemos a continuación el guión de las preguntas que se seguió con cada uno de los entrevistados: 1) Se puede presentar y decirnos cuál es su profesión actual. 2) ¿Cuál fue su relación con Patricia Stokoe? 3) ¿Qué recuerdos tiene de ella? 4) ¿Qué recuerda de la vida de Patricia Stokoe? 5) ¿Qué recuerda de la obra de Patricia Stokoe? 6) ¿Sabe algo de las influencias que tuvo Patricia en su vida y obra? 7) ¿Qué relación tiene Patricia Stokoe con la Expresión corporal en Argentina y en otros países? 8) ¿Qué aporta Patricia a la Expresión Corporal? 9) Quiere hacer un comentario final sobre Patricia Stokoe.

Hemos dividido el contenido del análisis de documentos y de las entrevistas personales en cuatro dimensiones/campos, y en ellas hemos establecido diferentes categorías, que a su vez y dependiendo del análisis del contenido, se subdividían en subcategorías. El tratamiento que hemos realizado de la información obtenida por los diferentes instrumentos utilizados, ha sido

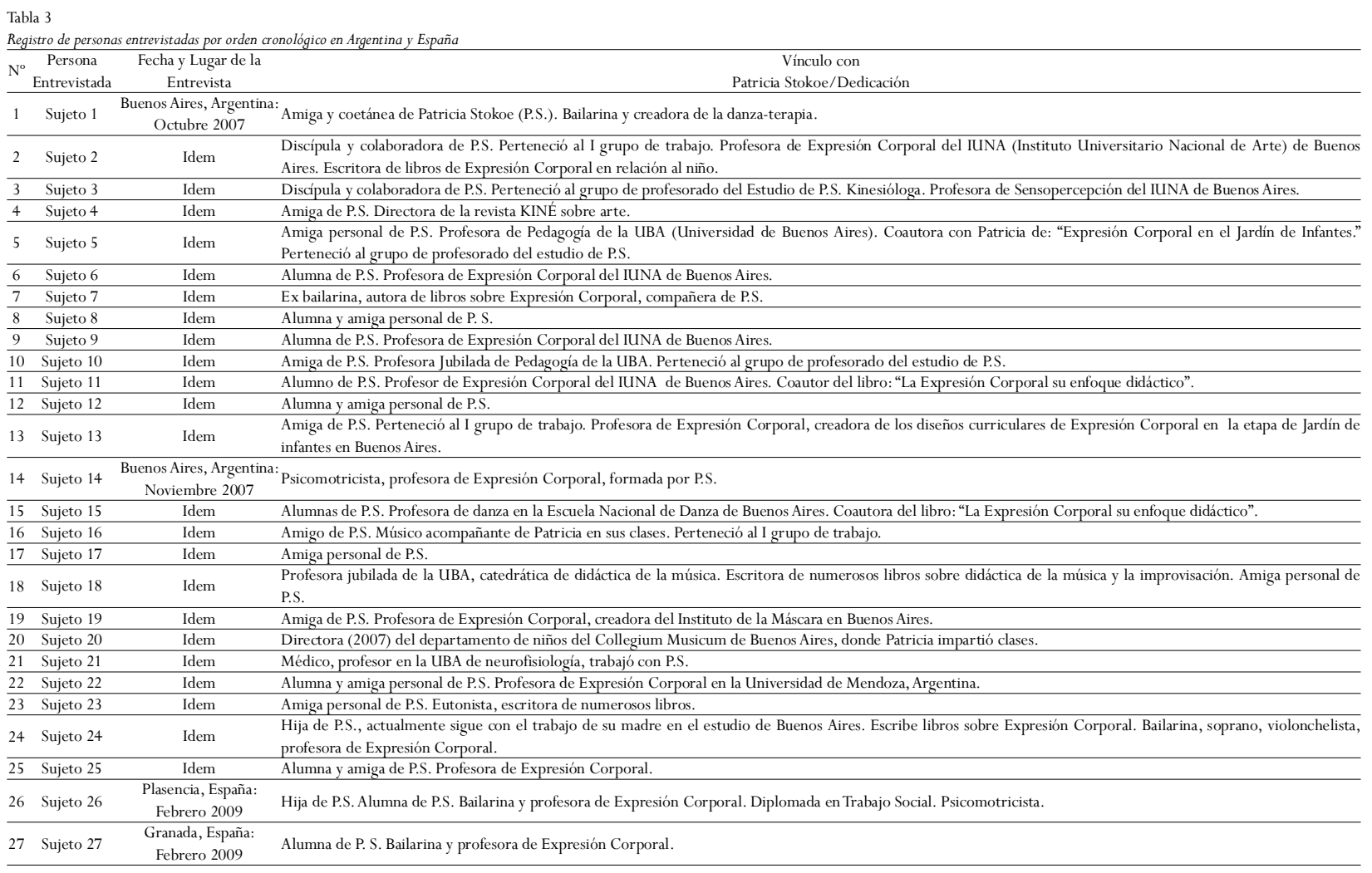


descriptivo transversal, incluyendo nuestra interpretación sugerida por el análisis de las diferentes entrevistas y por los documentos analizados que aportan información al campo de estudio.

Las dimensiones / campos que hemos establecido han sido: 1) Análisis de la vida de Patricia Stokoe (tabla 5). 2)Análisis de las influencias en la vida y la obra de Patricia Stokoe (tabla 6). 3) Análisis de la obra de Patricia Stokoe (tabla 7). 4) Revisión bibliográfica de la obra de Patricia Stokoe (tabla 8).

Tabla 5

Categorías y subcategorías: Análisis de la vida de Patricia Stokoe

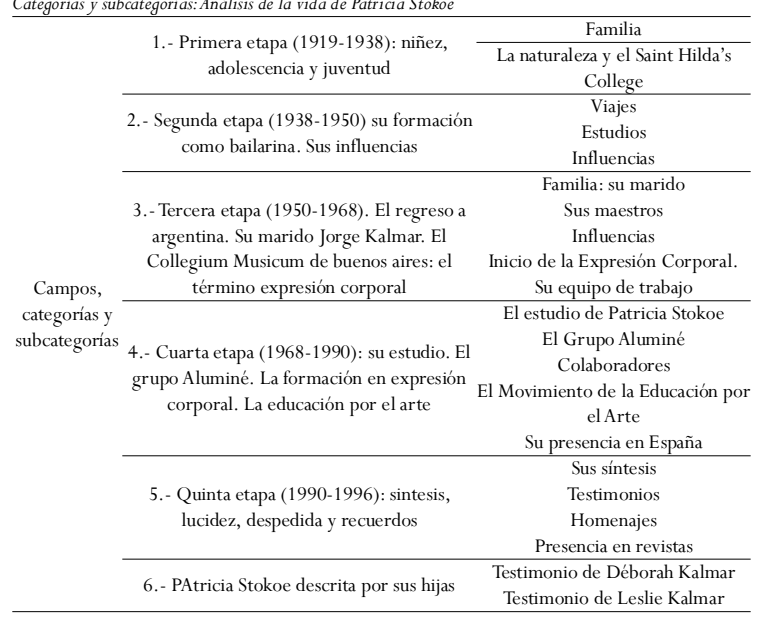

Tabla 6

Categorías y subcategorias: Análisis de las influencias en la vida y la obra de Patricia Stokoe 1. Influencias en la primera Familia.

etapa (1919-1938): niñez y Influencias Primeros años: la naturaleza y el Saint adolescencia y juventud. Hilda's College. Danza clásica en Inglaterra.

InfluenciasDanza libre de Laban, discípulos: Mille,

\begin{tabular}{ll} 
& \multicolumn{1}{c}{ directas. Jooss, Leeder y Ullmann. } \\
\cline { 2 - 2 } 2. Influencias en la segunda & Método Feldenkrais. \\
\cline { 2 - 2 } etapa (1938-1950): su & Ballet rusos de Diaghilev. \\
formación como bailarina. & Expresionismo alemán: Laban, Wigman,
\end{tabular} forman Sus influencias.

Influencias Jooss. Dalcroze.

indirectas. Delsarte y Noverre.

M. Gratericana:

M. Graham e I. Dunc
Método Alexander.

Jorge Kalmar.
Violeta de Gainza.

$\begin{array}{ll} & \text { Violeta de Gainza. } \\ \text { 3. Influencias tercera etapa } & \text { Gianni, Segal, Jaritonsky (primer grupo }\end{array}$ (1950-1967): el Collegium

Campos, Musicum de Buenos Aires: el directas. Oscar Fessler.

categorías y término Expresión Corporal. La Educación por el Movimiento: Irupe Pau y Fedora Abernstury.

Influencias La Bauhaus.

indirects Métodos musicales en Argentin Movimiento.

indirectas. ${ }^{K}$. Stanislawski.

4. Influencias cuarta etapa La danza en Argentina.

(1968-1990): el estudio de Gerda Alexander: la eutonía.

Patricia Stokoe. El grupo Otras personas: Sirkin, Rotbart, Nicosia

Aluminé. La formación en Influencias Otras pers

expresión corporal. La directas. Educación por el Arte en Latinoamérica

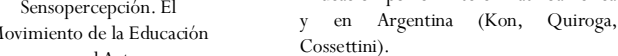
por el Arte.

5. Influencias en el epilogo

(1990-1996): síntesis, InfluenciasLiu Pai Lin (taichí).

lucidez, despedida y directas. Daniel Brenner recuerdos.

6. Influencias sobre la vida $y$

obra de Patricia Stokoe vista Testimonio de Déborah Kalmar. por su hija.

\section{Resultados}

Una vez realizada esta investigación pasamos a des-
Tabla 7

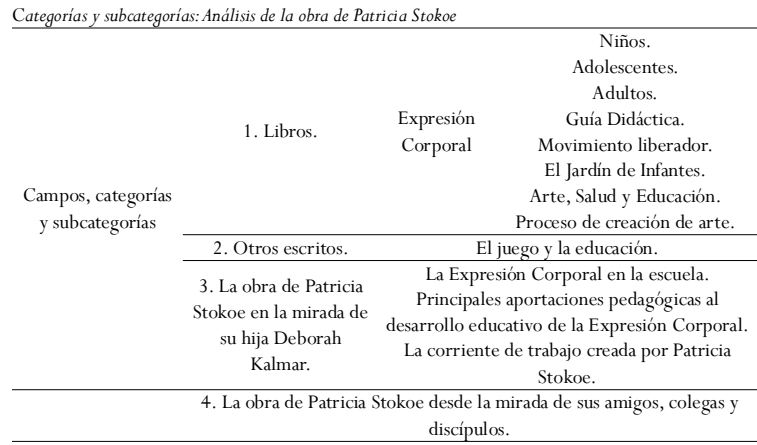

Tabla 8

\begin{tabular}{|c|c|}
\hline & $\begin{array}{l}\text { 1. Libros y estudios monográficos. } \\
\text { 2. Libros y estudios publicados en conjunto. } \\
\text { 3. Colaboración en obras de carácter general. } \\
\text { 4. Capitulos de libros. }\end{array}$ \\
\hline & $\begin{array}{l}\text { 5. Publicaciones diversas. Artículos en publicaciones periódicas. } \\
\text { Artículos en publicaciones no periódicas. }\end{array}$ \\
\hline & 6. Conferencias, ponencias, charlas. \\
\hline & 7. Talleres. \\
\hline & 8. Muestras / prácticas. \\
\hline Campos, categorías & 9. Clases. \\
\hline y subcategorías & $\begin{array}{l}\text { 10. Congresos. } \\
\text { 11. Jornadas. }\end{array}$ \\
\hline & 12. Encuentros. \\
\hline & 13. Cursos. \\
\hline & 14. Seminarios y mesas redondas. \\
\hline & 15. Simposium. \\
\hline & 16. Asociaciones de las que formó parte. \\
\hline & Entrevistas y reportajes. \\
\hline & notas periodísticas \\
\hline & notas periodisticas. $\quad$ Notas periodísticas sobre el grupo Aluminé. \\
\hline & 18. Creación artística con el Muestras de las obras. \\
\hline & grupo Aluminé. $\quad$ Estructuras con el grupo Aluminé. \\
\hline
\end{tabular}

cribir los resultados más relevantes obtenidos en relación los objetivos generales, centrándonos en el objetivo 2 :

1) Sobre el contexto histórico de Patricia Stokoe: El derrumbamiento de Europa, con las dos guerras mundiales, convulsionó la cosmovisión clásica. Se abrió una brecha a cuestionamientos vitales, sociales, políticos, artísticos, etc. Todos estos cambios influyeron en el continente latinoamericano. Éste fue arrastrado y conducido por las naciones hegemónicas e imperantes que son las que decidían a nivel mundial. En Argentina durante casi más de 50 años del siglo XX, no existió una democracia real. La revolución encumbró a líder tras líder, mandatario tras mandatario, a que dirigiera a un pueblo hastiado y dubitativo donde la violencia sería la respuesta.

Los cambios a nivel social son intensos y profundos e influyeron en todas las demás esferas. La mujer y su incorporación al mercado laboral, al mundo marcado territorialmente por el hombre, fueron los catalizadores de todo el proceso.

En el mundo de las artes explotaron los múltiples experimentos y se instalaron los mass media. Más que nunca el concepto de arte interacciona con la cultura y sociedad.Y, más que nunca, las artes (y las nuevas artes, producto de la tecnología creciente), se influyeron en- 
tre sí. La danza clásica se redefinió. Aparecieron la danza moderna y múltiples corrientes de tratamiento del cuerpo. Las escuelas gimnásticas interactuaron con las artes y con lo que la sociedad empezaba a demandar. El cuerpo es revalorizado y resituado, en una visión holística, primando la expresión, la unicidad, la creatividad, la comunicación y el placer. Aquellas artes susceptibles de acercamiento a cualquier persona se van concretando en los ámbitos artísticos, educativos y terapéuticos. Determinamos que unos de los momentos de inflexión a nivel mundial en todo lo comentado se tradujo en la década de los 60 con la explosión del mayo del 68 francés.

El hombre contemporáneo alineado en una sociedad de consumo, que antepone los valores materiales a la autorrealización personal y los sentimientos, dijo: ¡basta! El «cuerpo» con una nueva identidad resurgió. Así la EC fue fruto de su tiempo.

2) Sobre la vida de Patricia Stokoe: con respecto a la vida podemos concluir. La vida de Patricia Stokoe como la de muchas otras personas está encuadrada y marcada por los acontecimientos políticos, sociales, culturales y educativos por los que tuvo que transitar. De familia inglesa estuvo rodeada en su infancia por la naturaleza. Mujer de gran vitalidad pudo canalizar su energía en ese medio, obteniendo un gran dominio de su cuerpo. Sus padres la orientaban con unos estudios -en una escuela de tradición victoriana- para un modelo de vida. Ese no era su destino. Lo cambió. Decidió que quería bailar y viaja a Europa en 1938. (Figura 1)

Son las influencias de la ideología de RudolfVon Laban (Danza Libre o Free-Dance) y el trabajo de Moshé Feldenkrais quienes la hicieron plantearse decididamente su trabajo: ¿por qué seguir copias de modelos?, ¿puede ser uno mismo el modelo a seguir? Un desengaño amoroso grande, una enfermedad contraída de gira en el medio oriente, la necesidad de la luz, del sol de su tie-

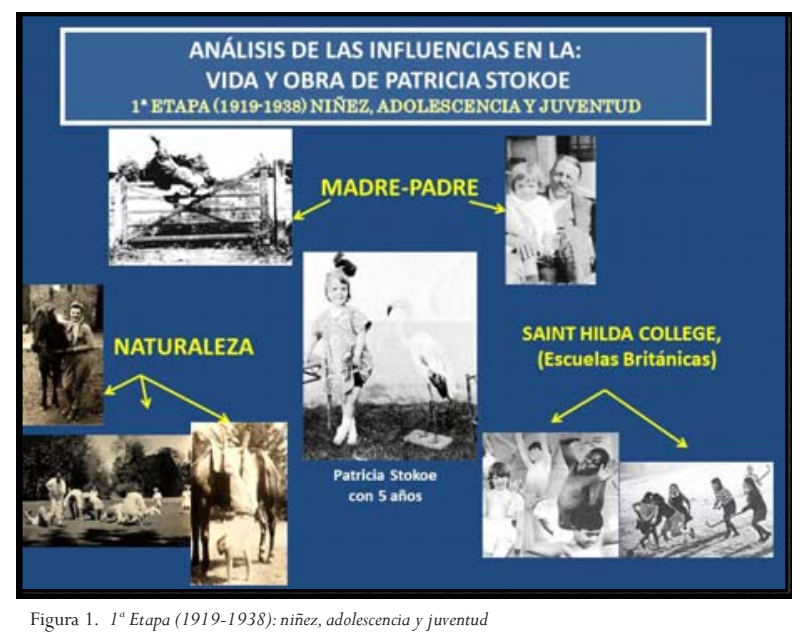

rra..., le hacen plantearse el regreso. Así en 1950 retornó a Argentina y allí conoció a Jorge Kalmar, arquitecto y músico que le puso en contacto con el Collegium Musicum (institución dedicada a la enseñanza de la música) de Buenos Aires. Allí desarrolló su quehacer, donde el trabajo de la danza estuviera al servicio de la enseñanza de la música para niños. Influida por Violeta de Gainza introdujo la improvisación en el movimiento corporal. Junto a las técnicas de incentivación teatral de Oscar Fessler, irá definiendo su trabajo. Fundamentales son los aportes de los músicos improvisadores Carlos Gianni y Eduardo Segal y de sus discípulos, destacando Perla Jaritonsky en su labor con niños. Se casa con Jorge Kalmar y fruto del amor nacen Deborah y Leslie Kalmar.

Su trabajo empezó a ser reconocido por los maestro/as jardinero/as que vieron una manera de encarar el trabajo corporal y musical en sus clases desde otra óptica. En este momento es cuando su labor alcanza una proyección mayor y comenzaron a hacerse formaciones en EC en el Collegium. Esta eclosión e interés por la actividad que desarrolló Patricia, le hizo plantearse que el cuerpo no debía estar supeditado al servicio de, sino que el cuerpo debía ser el eje, punto de partida y llegada de su trabajo. Toma contacto con las técnicas corporales de esos años (destacamos el sistema consciente de movimiento y la técnica Aberastury) que ella denominó: técnicas del movimiento. Estuvo siempre abierta a integrar en su trabajo las herramientas que las otras disciplinas le podían aportar. Así también entró en contacto con la tarea de dinámica grupal de Pichón Riviere y las fundamentaciones pedagógicas de Ruth Harf. Siempre defendió la identidad y los principios de lo que ella hacía, para no difuminarse o confundirse con otras disciplinas. Creó su estudio de trabajo en 1968 rodeándose de su equipo del Collegium Musicum. Comenzó aquí su formación en EC a nivel privado, entre 3 y 5 años, rodeándose de profesores de otras disciplinas afines.

Fue la directora y creadora del «Grupo Aluminé» donde desarrolló la creación colectiva para producir sus obras, método actualmente asumido pero hasta entonces no conocido. Generó en la Escuela Nacional de Danza una formación oficial y pública en EC, paralelamente a la formación pedagógica en su estudio a nivel privado. Con el paso de los años, en 1980, la EC fue reconocida e introducida en los planes de estudio públicos dentro de la formación del niño, siendo un triunfo personal.

$\mathrm{Su}$ primer equipo de trabajo (Gianni, Segal, Jaritonsky, Penchansky) se separaron en lo profesional de ella, aspecto que influiría anímicamente en Patricia pero que se sobrepondrá, ¡era una vikinga! Siguió ro- 
deándose de personas de distintas disciplinas que formaron su nuevo equipo de profesores en el estudio: educadores, artistas, kinesiólogos, psiquiatras, alimentándose así la EC y buscando de esta manera el cuerpo científico que Patricia siempre anheló para su disciplina.

El conocer a Gerda Alexander y su trabajo «la eutonía», hizo que Patricia Stokoe redefiniera su trabajo, marcando un punto de inflexión en su vida y obra. Creó la «sensopercepción» que se convirtió en método de conocimiento del cuerpo y del proceso creador para la EC. Se acercó y participó activamente en el «Movimiento de la Educación por el Arte» de manos deVíctor Kon y Sara Quiroga y conoció el trabajo de las hermanas Cossettini.

En los últimos años de su vida hubo una preocupación por generar una red de trabajadores sociales, donde los más desfavorecidos tuvieran también cabida. Que la EC verdaderamente fuera para todo el mundo. Fueron miles de alumnos los que disfrutaron de su maestría, tomando clases con ella en los cientos de cursos, conferencias, charlas, clases abiertas que impartió en Argentina y fuera de ella. Entre los países que impartió cursos: Guatemala, Nicaragua, Costa Rica, El Salvador, Panamá, Uruguay, Perú, Honduras, Brasil, Venezuela, Chile, Cuba entre otros. En España participó en la «Escuela de Psicomotricidad» de Barcelona desde 1988 a 1995 ininterrumpidamente.

3) Sobre la obra de Patricia Stokoe: Con respecto a la obra podemos concluir: evidenciamos varias etapas una vez analizada la obra de Patricia Stokoe.

$\varnothing \quad 1^{a}$ Etapa (1919-1938): Entusiasmada por la danza libre junto a otras disciplinas (moderna, comedia musical...) y sus incursiones en el método Feldenkrais, todo ello de su estancia en Londres (1938-1950), lo aplicó en el Collegium Musicum de Buenos Aires para niños, donde su trabajo estuvo supeditado a la música.

De esta etapa destacaron como los aspectos rítmicos estarían muy presentes en su obra. La improvisación musical con los aportes de Carlos Gianni y Eduardo Segal, serán una pieza fundamental en su obra. La estructura de las clases de danza profesor-pianista-alumno, Patricia la trastocó, ya que ella dejó que el músico interactuara mucho más en las clases desde su propia creatividad que estaba constantemente retroalimentándose con los alumnos y la profesora. En esta primera etapa tuvo peso específico la «imagen» (retratada por el fotógrafo Alex Shächter) y que aparecería en sus libros. Otra de las claves del quehacer de Patricia fue la integración de las técnicas de incentivación provenientes del teatro impartidas por Oscar Fessler.
Éste le encargó que impartiera en la Escuela Nacional de Teatro de Buenos Aires, la asignatura de cuerpo a Patricia Stokoe. Estas técnicas aplicadas a la danza son fundamentales y novedosas en la didáctica de la danza. La estimulación hablada como método en la danza.

Stokoe utilizó diferentes estímulos para incentivar a sus alumnos: la música, el habla (literatura, poesía...), la plástica, el uso de los objetos..., esto fue novedoso en la enseñanza de la danza, sobre todo en el desarrollo de los aspectos creativos y de comunicación. Así la aparición de imágenes mentales por el alumno para desarrollar estos aspectos. El uso del objeto fue fundamental en su obra: objeto que estimula a crear, a tomar conciencia del cuerpo, a relacionarme, a divertirme. El objeto adquiere significado relevante en la didáctica de Patricia.

Fruto de todo lo citado y al haber trabajado Patricia con niños en el Collegium, publicó sus dos primeros libros: «La Expresión Corporal y el niño» (1967) y «La Expresión Corporal y el adolescente» (1974). Más tarde publicó: «La Expresión Corporal» (1977). En este último libro era el adulto quien aparecía retratado por Alex Shächter. Stokoe que había empezado a desarrollar su trabajo con niños lo aplica también con los adultos: su método se abre camino.

El éxito de su trabajo y las primeras formaciones en EC llevó a nuestra autora a publicar: «Expresión Corporal. Guía didáctica para el docente» (1978). Recordamos que Patricia estuvo trabajando en una institución de enseñanza de la música, y su parte como educadora, estuvo presente en los tres libros anteriores y fue especialmente en la «Guía didáctica», donde estructura por primera vez de una forma clara sus concepciones sobre la EC (objetivos, contenidos, metodología, actividades). Creemos que este libro fue uno de los primeros manuscritos que pretendieron ordenar la disciplina de la EC. Entendió a la persona como una unidad: físico-psíquicoemocional, en donde se desarrollaba lo sensible, lo psíquico, lo motriz, lo creativo y lo social.

Ø $2^{a}$ Etapa (1938-1968): Fue en 1968 cuando Patricia se desató de las imposiciones exteriores (su éxito en el mundo de los adultos, con cursos que imparte por doquier que le permiten independizarse económicamente...) y centró su quehacer en el desarrollo propio de la disciplina EC. El cuerpo como eje central no supeditado. La EC como disciplina autónoma. Alentada en la formación que hizo a nivel privado en EC, integró especialistas de otras disciplinas: anatomía, educación, psicología..., buscando un cuerpo teórico-científico para la EC. Fundamental fue el aporte en dinámica grupal que ya venía implementando desde la primera etapa. 

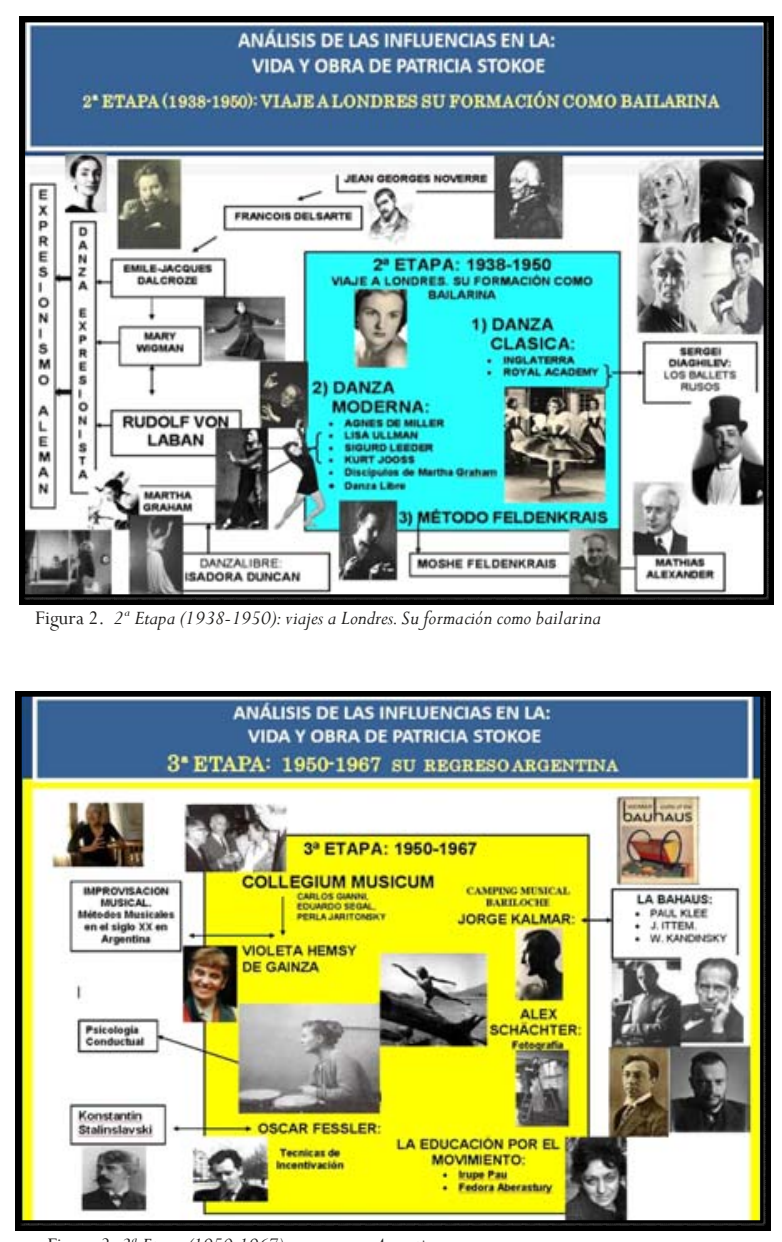

Era fundamental que el docente supiera contener y manejar al grupo. Éste debería formarse en estas técnicas. Patricia tomó contacto con la escuela de Pichón Riviere.

$\varnothing \quad 3^{a}$ Etapa (1968-1990): Punto de inflexión vital y que se vio reflejado en su obra fue la aparición de Gerda Alexander y la eutonía. El cuerpo entendido desde otra óptica, ya no desde un análisis anatómico, rítmico y de dinámica grupal que no por esto dejaban de estar presentes. La toma de conciencia del cuerpo, su cuidado, sus posibilidades, los procesos de creación que lleva a nivel mental por la incentivación hablada del docente, a imágenes productivas y reproductivas, fueron los ejes principales en esta etapa.

La EC creció y se transformó. Patricia desarrolló a partir de esto un trabajo que se convertiría en punto de salida y llegada para la propia EC. Nos referimos a la «sensopercepción» (técnica de base). Pensamos que Patricia aunque inició la sensopercepción no llegó a dar una explicación científica clara sobre ella. Fueron pocas las publicaciones que realizó en esta línea. Intentó dar explicaciones científicas, así sus encuentros con los doctores Cacurri y Berdichevsky pero pensamos que fueron sus discípulos quienes más lo investigarían en aras de ese cuerpo científico.

En 1979 es entrevistada por su amiga Violenta H. de Gainza, pedagoga musical y fruto de este encuentro se publica el libro: «Patricia Stokoe: diálogo con Violeta H. de Gainza».

Patricia en el grupo Aluminé investigó y desarrolló la sensopercepción, la creación colectiva y el trabajo con los objetos. En 1980 trabajó con Ruth Harf volviendo a otra etapa del niño en este caso, el jardín de infantes, publicando: «La Expresión Corporal en el Jardín de Infantes» (1980).

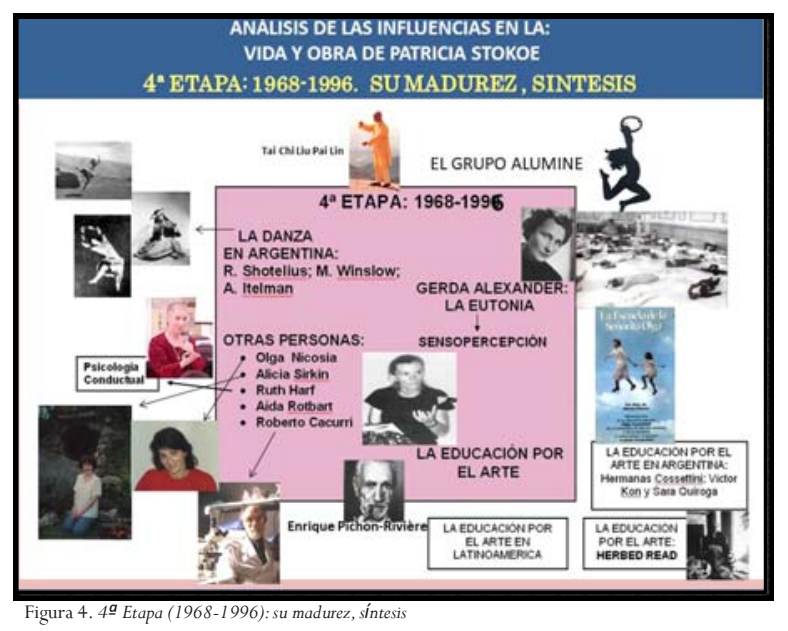

$\varnothing \quad 4^{a}$ Etapa (1990-1996): Patricia conoció el «Movimiento de la Educación por el Arte» por medio deVíctor Kon, Sara Quiroga y posteriormente al conocer la obra de las hermanas Cossettini. La antigua y limitadora definición de educación para el arte, pasa a transformarse en educación por el arte.

En 1987 en el libro: «Expresión Corporal: Arte-Salud-Educación», Patricia reunió conferencias, jornadas, charlas... que había impartido a lo largo de años. La EC se ubicó en esta tríada, de la cuál sería germen de las actuales concepciones. Si Rudolf Laban promulgó una danza para una sociedad libre, la educación por el arte colocó a la danza como eje principal de la sociedad. Hubo una preocupación ideológica de Patricia de poner la danza, el arte al servicio de todos, donde todos éramos iguales: ¿utopía? Junto a su amiga Alicia Sirkin en 1987 escribió el libro: «El Proceso de Creación en el Arte», intentando buscar ese método creativo clave en los procesos artísticos.

El trabajo con los desfavorecidos, los niños de la calle, marginados fueron su preocupación en esta etapa. Sus esfuerzos se encaminaron en la construcción de una red de trabajadores sociales y en la conferencia de Japón de 1990, habló de los niños de la calle de su país. Siguió haciendo que la EC siguiera creciendo con sus incursiones en técnicas orientales como el taichí. 
La obra fue continuada por sus hijas: Déborah y Leslie Kalmar. Déborah continuó con el estudio de trabajo de Patricia en Buenos Aires (Estudio Kalmar Stokoe). Imparte cursos por toda Latinoamérica y Europa. Redefinió el trabajo de su madre. Así denomina en tres áreas los pilares de la EC: A) El área del Cuerpo y el Movimiento: a.- Sensopercepción, motricidad y tono. b.- Espacio. c.- Calidades de movimiento. d.Corporización de elementos de la música. B) El área de la Comunicación. C) El área de la Creatividad.

\section{Conclusiones}

Una vez contrastadas las opiniones de las entrevistas y analizados los constructos de las respuestas podemos concluir que Patricia Stokoe fue una de las pioneras en la construcción y génesis de la expresión corporal.

Patricia Stokoe pertenece a un grupo de mujeres que con el ejemplo de su vida y sin ella ser consciente, colocan a su género en el sitio que le corresponde. Lo cual histórica, cultural y socialmente no siempre había sido así. Hombres y mujeres, con sus maravillosas diferencias, caminan juntos para construir una sociedad más digna, más libre, más humana. El ideal humanista se convierte en hechos desde esta nueva concepción del cuerpo, de educación por el arte. El siglo XX es el «Siglo de la Mujer», el siglo de las mujeres viajeras, valientes, decididas, seguras, luchadoras, sensibles que deben adaptarse a seguir teniendo los mismos roles de antaño: esposa y madre, pero que además deben integrarse en el mundo laboral. Esta revolución constituye uno de los cambios sociales más trascendente del siglo XX.

Patricia, artista y bailarina, busca más allá de la copia del modelo que la danza clásica y la moderna predican. Ella lo hace desde su ejemplo, rompiendo moldes. Es una de las primeras maestras que se atreven a impartir clase embarazada: el cuerpo siempre presente, sin avergonzarse de él.Y será de las primeras maestras que mezcle técnicas de otras artes, así utilizará las «técnicas de incentivación» teatrales. También, posibilitará al músico pianista a que improvise, cree y genere en sus clases: la música se hace fundamental en su quehacer. Confianza y fusión determinan su obra: libertad como responsabilidad creadora.

Patricia Stokoe hace algo que es denominador común a las Artes en el siglo XX: las acerca al pueblo. La antigua sociedad clasista, donde las artes pertenecían a unos pocos afortunados, se desmorona. En una sociedad donde el arte se está haciendo cada vez más tecnológico y omnipresente, Patricia vuelve al cuerpo, lo hace cotidiano, lo hace para todos. $\mathrm{Al}$ acercar el arte al pueblo: «la danza de cada uno», cada individuo es responsable, haciéndose su agente creador su propio maestro. Patricia está desatando imposiciones que vienen del exterior, en definitiva hace al ser humano más libre.

En la propuesta de Patricia Stokoe subyace un ser humano que elige desde su libertad y realidad, pudiendo llegar a construir su vida. Se cuestiona: si puedo crear mi propia danza, ¿puedo ser dueño de mi propia vida?; ¿puedo ir construyendo mi propio destino? Destino en el que no se obvia a la sociedad pero que no decide por él. Lo lúdico, el placer del juego, el placer de sentir el propio cuerpo, de estar relacionándose, el placer de la propia piel, la alegría de vivir, la creación... van a estar presentes en su trabajo. ¿Por qué renunciar a ellos? Se acabaron las imposiciones de antaño. La toma de consciencia y conciencia del «ser» se hace desde un sentido holístico. El cuerpo se hace eje vertebrador de esta revolución de dentro afuera. Considera y entiende a un ser humano desde una perspectiva, ética y de igualdad donde todos tienen la capacidad de comunicarse y crear. El ser humano como obra de arte, siendo cada sujeto único e irrepetible.

Insistimos, la Expresión Corporal es fruto de su tiempo y Patricia Stokoe una de sus «parteras». Resaltamos estas seis razones:

1. Defendió una actividad democrática, el arte para todos, sin encasillarse en elitismos que la hubieran desbancado enseguida. 2. Promulgó el sentido ético de la actividad: una danza propia, para la libertad y la igualdad, para la vida. 3. Desarrolló su actividad fusionando y recibiendo de artistas, pedagogos y corporalistas. Promovió que la búsqueda fuera compartida: creía en las redes y las creó. 4. Intentó estructurar su tarea con anclajes científicos y para ello escribió sus libros. Destacamos que sin las publicaciones, se podría haber diluido en la profusión de teorías de la época. 5. Se mantuvo en el trabajo: ¡treinta y dos años en su estudio! Así consiguió ser un referente incuestionable: por dedicar toda una vida profesional a desarrollar y enriquecer una tarea a largo plazo, y por formar a cientos profesionales. 6. Su gran aportación, además de trabajar fuera del sistema con las dificultades políticas y económicas que tuvo que sortear, fue entrar en el sistema: consiguió un título oficial, el reconocimiento del Profesorado Nacional en 1980.

Pensamos que estos principios, esta historia -su historia-, debe ser conocida especialmente por los futuros docentes que tendrán que impartir la EC en los Cole- 
gios e Institutos. En una disciplina emergente como es la EC, pensamos que este trabajo, junto a otros que se están desarrollando, pueden aportar «corpus teórico» tan necesario en los comienzos.

\section{Referencias}

Abilleira, M. y Fernández, M. (2017). Análisis de la formación del maestro/a de educación infantil en expresión corporal. Emasf. Revista Digital de educación Física, 9 (49), 36-59.

Aguirre, S. (1995). The professional stranger. An informal introduction to ethnography. NuevaYork, United States: Academic Press.

Armada, J., González, I. y Montávez, M. (2013). La expresión corporal: un proyecto para la inclusión Corporal. Retos. Nuevas tendencias en Educación Física, Deporte y Recreación, 24, 107-112.

Arroyo, R. (2005). Enseñanza de la Meta cognición de la composición escrita en contextos Multiculturales. Educación, Desarrollo y Diversidad, Vol. 8 (3), 31-50.

Baena, A. Granero, A. y Gómez, M. (2009). La epistemología de la Educación Física en relación al currículum de Secundaria en la Ley orgánica de Educación. Retos. Nuevas tendencias en Educación Física, Deporte y Recreación 15, 49-56.

Buendía, L., Colas, P. y Hernández, F., (1998). Métodos de investigación en Psicopedagogía. Madrid: McGrawHill.

Cachadiña, M. (2004). Expresión Corporal y creatividad: Métodos y procesos para la construcción de un lenguaje integral. Tesis Doctoral. Universidad Politécnica, de Madrid. Madrid.

Calvo, A., García, I. y Pérez, R. (2013). Expresión corporal. Una práctica de intervención que permite encontrar un lenguaje propio mediante el estudio y la profundización del empleo del cuerpo Retos. Nuevas tendencias en Educación Física, Deporte y Recreación, 23, $19-22$.

Cardona, A. (2009). Patricia Stokoe: vida y obra. La creadora de la Expresión Corporal-danza. Tesis doctoral. Universidad de Granada. Granada.

Cardona, A. y Kalmar, D. (2011). La vida de Patricia Stokoe. Sevilla, España: Wanceullen.

Cardona, A. (2019). Análisis de la vida y la obra de Patricia Stokoe desde la mirada de sus amigos, colegas y discípulos. En F. Ruiz, F. González \& A. Calvo. (Eds.). XIII Congreso Internacional FEADEF sobre la enseñanza de la Educación Física y el deporte escolar II Congreso Red Global (pp.569-582). FEADEF
Cohen, L. y Manion, L. (1990). La entrevista. En Cohen, L. y Manion, L. Métodos de investigación educativa. Madrid, España: La Muralla.

Colás, M. y Buendía, L. (1994). Investigación Educativa. Sevilla: ALFAR.

Coterón, J. y Sánchez, G. (2012) Expresión corporal en Educación Física: la construcción de una disciplina. Emasf. Revista Digital de educación Física, 3 (14), 164175.

Coterón, J. y Sánchez, G. (2013) Expresión Corporal en Educación Física: bases para una didáctica fundamentada en los procesos creativos. Retos. Nuevas tendencias en Educación Física, Deporte y Recreación, 24, 117 122.

De la peñas, E. y Gil, J. (2019). Expresión corporal y discapacidad intelectual ligera. Una propuesta de adaptación ligera. Emasf. Revista Digital de educación Física, 10 (56), 104-118.

Denzin, N. K. \& Lincoln,Y. (2000). Handbook of qualitative research. London's: Sage.

García, A. (2015). Encuentro con Patricia Stokoe. Buenos Aires, Argentina: Autoedición.

Gillham, D. (2000). Case study research methods. London y New York: Continuun

Goezt, J. y Lecompte, M. (1988). Etnografía y diseño cualitativo en investigación educativa. Madrid: Morata.

Harf, R. y Stokoe, P. (1980). La Expresión Corporal en el Jardín de Infantes. Buenos Aires, Argentina: Paidós.

Jones, E. (1985). Manual for the Psychotherapy Process Qset. Manuscrito no publicado. Berkeley, CA. University of California.

Kalmar, D. (2003). Con los ojos del corazón. En G. Sánchez \& F. J. Coterón (Comps). Expresión, Creatividad y Movimiento I Congreso Internacional de Expresión Corporal y Educación (pp. 31-48) Salamanca: Amaru.

Kalmar, D. (2005). Qué es la Expresión Corporal. A partir de la corriente de trabajo creada por Patricia Stokoe. Buenos Aires, Argentina: Lumen.

Kalmar, L. (2003). Patricia Stokoe y la Expresión Corporal. En G. Sánchez \& F. J. Coterón (Comps). Expresión, Creatividad y Movimiento. I Congreso Internacional de Expresión Corporal y Educación. (pp. 1730). Salamanca: Amaru.

Latorre, A., Del Rincón, D. y Arnal. J. (2003). Bases metodológicas de la Investigación Educativa. Barcelona: Experiencia.

Mercado, F.J. (2000). El proceso de análisis de los datos en una investigación sociocultural en salud. En F. J. Mercado y T. M. Torres (Comps). Análisis Cualitativo 
en Salud. Teoría, Método y Práctica. México: Universidad de Guadalajara.

Miles, M. B. \& Hunerman, A. M. (1994). Qualitative data analysis $\left(2^{\circ} \mathrm{Ed}\right.$.). Thousand Oaks, CA: SAGE.

Montávez, M. (2012). LOE. La consolidación de la expresión corporal. Emasf, Revista Digital de Educación Física (3)14, 60-80.

Padilla, C., Pérez, C., Fernández, B. (2012). La Expresión Corporal en la enseñanza universitaria/Galo Sánchez y Javier Coterón (Eds.). Salamanca: Ediciones Universidad Salamanca.

Palomares, J. (2003). Motivaciones, hábitos y usos de los espacios del Parque periurbano "Dehesas del Generalife» de Granada. Tesis Doctoral no publicada. Departamento de Didáctica de la Expresión Musical, Plástica y Corporal. Universidad de Granada.

Peña, V. y Vicente G. (2019). Danza en educación infantil: opinión de los docentes. Retos. Nuevas tendencias en Educación Física, Deporte y Recreación, 36, 239-244.

Ruano, M. (2004). La influencia de la Expresión Corporal sobre las emociones: Un estudio experimental. Tesis Doctoral, no publicada. Departamento de Física e Instalaciones aplicadas a la Edificación al Medio Ambiente y al Urbanismo de la Escuela Técnica Superior en Arquitectura. Universidad Politécnica de Madrid.

Ruano, M. y Sánchez, G. (2009). Expresión Corporal y Educación Sevilla, España: Wanceullen.

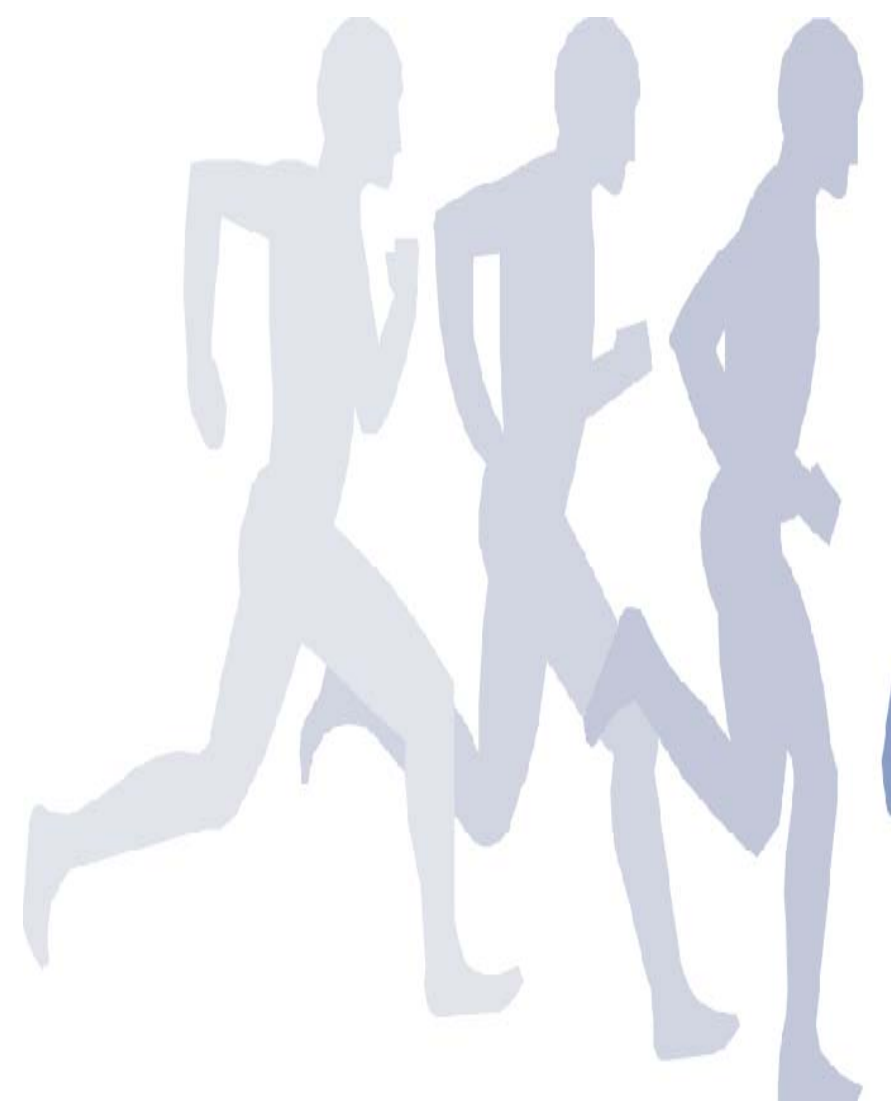

Santos, M. A. (1990). Hacer visible lo cotidiano. Madrid: Editorial Akal. Sirkin, A. y Stokoe, P. (1994). El Proceso de creación en el arte. Buenos Aires, Argentina: Almagesto.

Spradley, J. (1979). The Ethnographic Interview. New York. Stake, R. (1998). Investigación con estudio de caso: Barcelona: Morata.

Stokoe, P (1967). La Expresión Corporal y el Niño. Buenos Aires, Argentina: Ricordi.

Stokoe, P (1974). La Expresión Corporal y el Adolescente. Buenos Aires: Barry.

Stokoe, P. (1978). Expresión Corporal. Guía didáctica para el docente. Buenos Aires, Argentina: Ricordi Americana.

Stokoe, P. (1987). Expresión Corporal. Arte, Salud y Educación. Buenos Aires, Argentina: Humanitas.

Taylor, S. y Bogdan, R. (1987). Introducción a los métodos cualitativos de in-vestigación. La búsqueda de significados. Barcelona: Paidós.

Torres, B. (2008). Conocimientos, pensamientos, creencias y teorías implícitas del profesorado de Educación Física acerca de las aportaciones didácticas de la obra del pedagogo Francisco Amorós al desarrollo de la Educación Física moderna. Tesis Doctoral, no publicada. Departamento de Didáctica de la Expresión Musical, Plástica y Corporal. Universidad de Granada.

Visauta, B. (1989). Técnicas de investigación social. Barcelona: PPU.

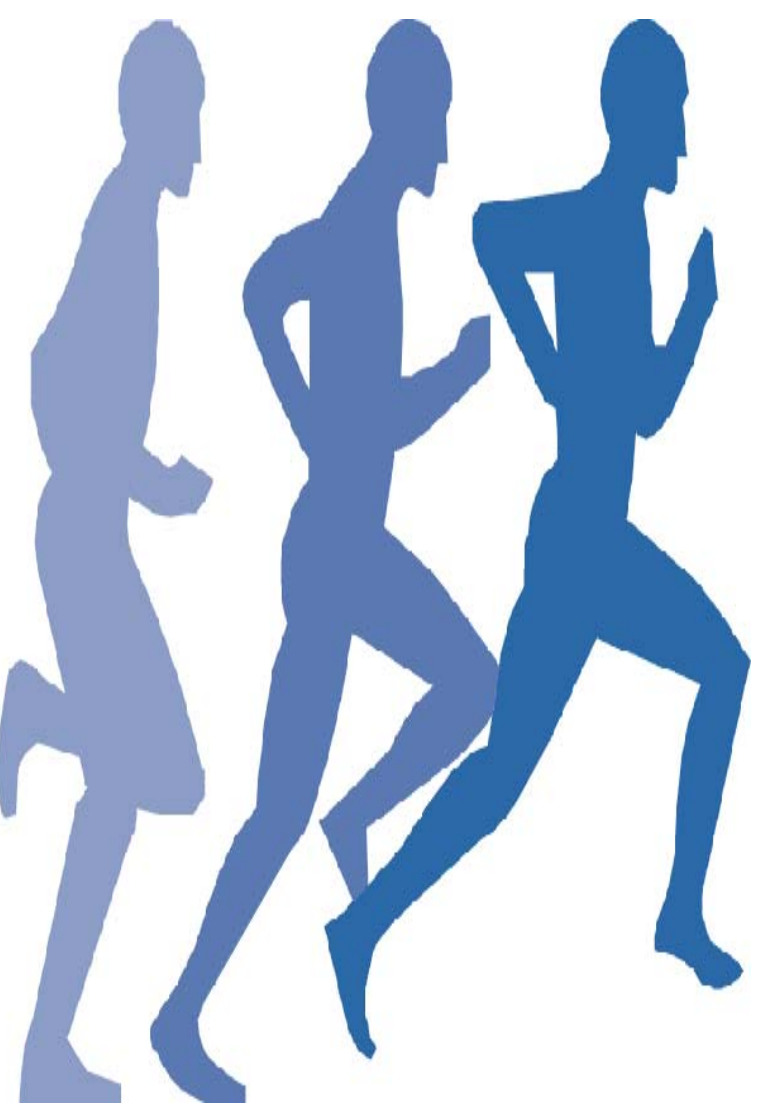

